\title{
Consultation-Liaison Psychiatry for Outpatients in a Great Metropolitan Hospital
}

\author{
Matteo Porcellana, Roberta Bassetti, Adelaide Panariello, Mauro Percudani \\ ASST Great Metropolitan Hospital Niguarda, Milan, Italy
}

\begin{abstract}
Consultation-liaison psychiatry remains the guardian of holistic approach to the patient and consultation services in general hospital could therefore be referred to as "filters" for mental health care, in particular for patients with minor depressive or anxiety disorders. It is worth noting that psychiatric disorders, even when sub-clinical, worsen outcome whereas their early detection and treatment reduce health care costs significantly. The present study was transversal, exploratory, and descriptive in order to investigate the sample of outpatients referred from general practitioners (GP) to service of consultation-liaison psychiatry of the ASST Great Metropolitan Hospital Niguarda of Milan over a 7-months period. Data from all psychiatric consultations for outpatients were collected by a structured clinical report including socio-demographic features, features of referrals, and features of back-referrals. Five hundred and eleven consultations were performed. Participants were mainly female (58.7\%) with an average age between 45 and 55 years (27.6\%); 31.1\% of these patients was at the first contact with a mental health specialist. The diagnoses formulated were distributed as follows: The most common diagnoses were affective disorders $(44.6 \%)$, followed by neurotic disorders $(34.1 \%)$, personality disorders $(6.8 \%)$, and finally, psychosis (6.2\%). The study showed an example of a consultation-liaison service situated in a Great Metropolitan Hospital offering to GP the opportunity of a diagnostic assessment and shared management of minor psychiatric disorders. This approach could lead to several vantages, such as the management in a non-psychiatric setting of patients with reduction of stigma and reduced costs for specific psychiatric services where are referred more severe patients.
\end{abstract}

Keywords: consultation-liaison psychiatry, primary care, minor depressive and anxiety disorders

\section{Background}

In Italy, the 1978 legislation stated a general guideline but did not provide specific directions as to its implementation; it left Italian regions responsibility for managing the transition toward community psychiatric care and a more recent legislation has again emphasized the role of the regions in planning, coordination, and delivery of health care services.

Lombardy is the largest and the most affluent region in Italy. It is located in Northern Italy and includes

Matteo Porcellana, MD, MSc, Psychiatry, Department of Mental Health and Dependencies, ASST Great Metropolitan Hospital Niguarda, Milan, Italy.

Roberta Bassetti, MD, Psychiatry, Department of Mental Health and Dependencies, ASST Great Metropolitan Hospital Niguarda, Milan, Italy.

Adelaide Panariello, MD, Psychiatry, Department of Mental Health and Dependencies, ASST Great Metropolitan Hospital Niguarda, Milan, Italy.

Mauro Percudani, MD, Psychiatry, Director of the Department of Mental Health and Dependencies, ASST Great Metropolitan Hospital Niguarda, Milan, Italy. 
the metropolitan area of Milan, the second largest Italian city. Patients can access specialist mental health (MH) services directly, although they are more frequently referred by their primary care physicians.

Since the first Regional Mental Health Plan approved in the early 1980, Lombardy region has developed a network of Community Mental Health facilities and adopted an organizational model for Department of Mental Health based on multi-professional teams.

The Regional Plan approved in 2004 prompted the development of clinical pathways within Community Mental Health Centres with a view to achieving a more appropriate differentiation of clinical pathways between patients with severe mental illness and those with common mental disorders. In this view, for users with common mental disorders who do not need specialized care, it was suggested counselling with General Practitioners (Lora, Barbato, Cerati, Erlicher, \& Percudani, 2012; Lora, Starace, Di Munzio, \& Fioritti, 2014).

General practitioners are confronted daily by physical/psychiatric multimorbidity with its extensive costs of suffering for patients and consumption of medical and economic resources (Menchetti, Tarricone, Bortolotti, \& Berardi, 2006; Smith, 2009).

It is worth noting that psychiatric disorders, even when sub-clinical, worsen outcome whereas their early detection and treatment reduce health care costs significantly (De Giorgio et al., 2015; Ormel et al., 1999).

Lipowsky (1983) defined consultation-liaison psychiatry (CLP) as "a sub-specialisation of psychiatry focusing on diagnosis, treatment, study and prevention of psychiatric morbidity in patients with organic pathologies and those with somatisation" (p. 329).

In this same sense, Ajiboye (2007) identified CLP as "The guardian of holistic approach to the patient" (p. 201), underlining its pre-eminent role in management who are admitted to a general hospital.

Consultation services in general hospital could therefore be referred to as "filters" for mental health care, in particular for patients with minor depressive or anxiety disorders (Burian, Franke, \& Diefenbacher, 2016).

\section{Method}

The present investigation is a non-experimental, cross-sectional, exploratory, and descriptive study. Data from all psychiatric consultations for outpatients referred from general practitioners (GP) to service of consultation-liaison psychiatry of the ASST Great Metropolitan Hospital Niguarda of Milan over a 7-months period (2018) were collected by a structured clinical report including socio-demographic features, features of referrals, and features of back-referrals.

\section{Results}

During the study period, 511 consultations were performed. There were 300 women (58.7\%) and 211 men (41.3\%); $27.6 \%$ of the subjects were aged $45-55 ; 26.6 \%$ over 65 years old; $22.9 \%$ were $56-65$ years old; $10.6 \%$ were 35-44 years old; $9.6 \%$ were $25-34$ years old; and $2.7 \%$ were under 25 years old.

Half of the sample $(N=234,47.1 \%)$ were married, $31.8 \%$ had never been married, $4.8 \%$ were widowed, and $16 \%$ were divorced/separated; $40.9 \%$ were employed, $27.1 \%$ retired; $6.6 \%$ were housewives, and $16.9 \%$ were unemployed.

Education status was as follows: $50(10 \%)$ had received less than eight years, $160(32.1 \%)$ had received eight years, $212(42.5 \%)$ had received 13 years, and 77 (15.4\%) had received 18 years.

In $98 \%$ of cases, an ICD-10 diagnosis was made by consultants and in $2 \%$ no psychiatric diagnosis was made. A diagnosis of neurotic, stress-related, and somatoform syndromes (F40-48) was made in $34.1 \%$ of 
participants $(N=170)$, affective syndromes (F30-39) were diagnosed in $44.6 \%$ of patients $(N=222)$. Organic mental syndromes (F00-09) were found in $1.4 \%(N=7)$ and another $3.8 \%(N=19)$ were given a primary diagnosis of mental and behavioural disorders due to the use psychoactive substances (F10-19). Schizophrenia, schizotypal, and delusional disorders (F20-29) were reported in 6.2\%; personality disorder in $6.8 \%$; and in $0.6 \%(N=3)$ a diagnosis of behavioural syndromes associated with physiological disturbances and physical factors (F50-59).

\section{Discussion and Conclusion}

Implementation of consultation-liaison psychiatry (CLP) services within family medicine has been already reported by other Italian studies (Ferrari \& Rigatelli, 2006; Menchetti et al., 2006) with data showing the efficacy of the collaboration between psychiatrists and general practitioners working in the community.

Some data are also available in Italy regarding the role of CLP services and CLP educational intervention in training physicians, psychiatrists, and specialists in other branches of medicine, and nurses working in the general hospital (Rigatelli, Ferrari, Uguzzoni, \& Natali, 2000).

However, some problems are still unresolved, including lack of funding to formally integrate plans of cooperation between general hospital, community medicine, and CLP; heterogeneity of experiences and activities within CLP; the need to improve standards of clinical practice, clinical management, training; and, most important, the need for research on CLP (Ferrari \& Rigatelli, 2006; Grassi et al., 2015).

This study was conducted with the aim to investigate the characteristics of a sample of outpatients referred from general practitioners to the service of consultation-liaison psychiatry of the ASST Great Metropolitan Hospital Niguarda in Milan.

Regarding the specific features of referred patients, most were women (58.7\%), aged 45-65, married, employed, and with a medium educational level. These results are specific of our sample and differ from those reported in other studies showing that Consultation-Liaison services are most frequently offered to the most vulnerable segments of population such as very elderly and belonging to a socioeconomic low class (Huyse et al., 2001).

In $98.8 \%$ of cases, the psychiatrist made an ICD-10 diagnosis, with percentages comparable with those found in other studies (Gala et al., 1999; Hengeveld, Rooymans, \& Vecht van den Bergh, 1984; Huyse et al., 2000, 2001). Affective disorders were diagnosed in $45 \%$ of the patients, followed by neurotic, stress-related disorders, and somatoform disorders. Certain groups of diagnosis were less frequent in our sample than in other investigations; for example, a diagnosis of mental or behavioural disorder due to the use of psychoactive substances was infrequent (3.8\%) in this population compared to the highest rates found in other studies (Poli et al., 2017) suggesting that general practitioners refer these patients to specific services, such as for dependence ones.

According to the obtained results, it is observed that anxiety and depressive disorders occur frequently in primary care; several studies showed that patient management in this setting in often inadequate (Robinson, Geske, Prest, \& Barnacle, 2005; von Koff, 2001) and that these disorders are associated with high degrees of subjective distress and disability (Cujipers \& Smit, 2002; Ustun, Ayuso-Mateos, Chatterji, Mathers, \& Murray, 2004) and have heavy social costs (Lokkerbol, Weehuizen, Mavranezouli, Mihalopoulous, \& Smit, 2014).

These results confirm that the, e.g., "minor" psychiatric disorders may represent an important public health problem which involves not only mental health services, but primary care as well since many patients suffering 
from these disorders are seen and managed there. This observation points out the need of a strong cooperation between primary care and psychiatric services.

In 1999, the Italian National Health care system began to encourage an integration between primary and secondary care for the management of mental health, subsequently mandating that the Community Health Centres develop primary care consultation-liaison services. More recently, a Regional Law (L23/2015) in Lombardy has considered crucial for a good organisation of health services to establish a continuity between general hospitals and primary care services, particularly for chronically ill patients.

Our study showed an example of a consultation-liaison service situated in a big general hospital offering to general practitioners the opportunity of a diagnostic assessment and shared management of minor psychiatric disorders. This approach could lead to several vantages, such as the management in a non-psychiatric setting of patients with reduction of stigma and reduced costs for specific psychiatric services were are referred more severe patients.

Despite its limits, such as the naturalistic design, the small number of the sample, and the short period of observation, our study described a consultation-liaison psychiatry experience which could provide a model for implementing this kind of approach to minor psychiatric disorders in general hospital.

\section{References}

Ajibove, P. O. (2007). Consultation-liaison psychiatry: The past and the present. African Journal of Medicine and Medical Sciences, 36, 201-205.

Burian, R., Franke, M., \& Diefenbacher, A. (2016). Crossing the bridge-A prospective comparative study of the effect of communication between a hospital based consultation-liaison service and primary care on general practitioners' concordance with consultation-liaison psychiatrists' recommendations. Journal of Psychosomatic Research, 86, 53-59.

Cujipers, P., \& Smit, F. (2002). Excess mortality in depression: A meta-analysis of community studies. Journal of Affective Disorder, 72(3), 227-236.

De Giorgio, G., Quartesa, R., Sciarma, T., Giulietti, M., Piazzoli, A., Scarponi, L., ... Piselli, M. (2015). Consultation-liaison psychiatry-from theory to clinical practice: An observational study in general hospital. BMC Research Notes, 8, 475. doi: 10.1186/s13104-015-1375-6

Ferrari, S., \& Rigatelli, M. (2006). General Hospital psychiatry in Italy: An update. Journal of Psychosomatic Research, 60, $217-218$.

Gala, C., Rigatelli, M., De Bertolini, C., Rupolo, G., Gabrielli, F., \& Grassi, L. (1999). A multicenter investigation of Consultation-liaison psychiatry in Italy. General Hospital of Psychiatry, 21, 310-317.

Grassi, L., Mitchell, A. J., Otani, M., Caruso, R., Nanni, M. G., Hachizuka M., ... Riba, M. (2015). Consultation-liaison psychiatry in the general hospital: The experience of UK, Italy, and Japan. Current Psychiatry Reports, 17, 44. doi: 10.1007/s11920-015-0581-1

Hengelveld, M. W., Rooymans, H. G., \& Vecht van den Berg, R. (1984). Psychiatric consultations in a Dutch university hospital: A report on 1814 referrals, compared with a literature review. General Hospital of Psychiatry, 6(4), 271-279.

Huyse, F. J., Herzog, T., Lobo, A., Malt U. F., Opmeer, B. C., Stein, B., ... Tienari, P. (2000). European Consultation-liaison psychiatric services: The ECLW collaborative study. Acta Psychiatrica Scandinavica, 101(5), 360-366.

Huyse, F. J., Herzog, T., Lobo, A., Malt U. F., Opmeer, B. C., Stein, B., ... Tienari, P. (2001). Consultation-liaison psychiatric service delivery: Results from a European study. General Hospital of Psychiatry, 23(3), 124-132.

Lipowsky, Z. J. (1983). Current trends in consultation-liaison psychiatry. Canadian Journal of Psychiatry, 28(5), 329-338.

Lokkerbol, J., Weehuizen, R., Mavranezouli, I., Mihalopoulous, C., \& Smit F. (2014). Mental health care system optimization from a health-economics perspective: Where to sow and where to reap? Journal of Mental Health Policy and Economy, 17(2), 51-60.

Lora, A., Barbato, A., Cerati, G., Erlicher, A., \& Percudani, M. (2012). The mental health system in Lombardy, Italy: Access to services and patterns of care. Social Psychiatry and Psychiatric Epidemiology, 47, 477-454.

Lora, A., Starace, F., Di Munzio, W., \& Fioritti, A. (2014). Italian community psychiatry in practice: Description and comparison of three regional systems. The Journal of Nervous and Mental Disease, 202, 446-450. 
Menchetti, M., Tarricone, I., Bortolotti, B., \& Berardi, D. (2006). Integration between general practice and mental health services in Italy: Guidelines for consultation-liaison services implementation. International Journal of Integrated Care, 6. Retrieved from http://www.ijic.org

Ormel, J., Vonkorff, F., Oldenhinkel, A. J., Simon, G., Tiemens, B. G., \& Ustun, T. B. (1999). Onset of disability in depressed and non-depressed primary care patients. Pscyhological Medicine, 29, 847-853.

Poli, R., Carreca, A., Colmegna, F., Ferraris, S., Gagliardi, E., Tamburini, S., \& Toscano, M. (2017). The practice of consultation psychiatry in Italy: a multi-centre study. Journal of Psychosomatic Research, 96, 32-34.

Rigatelli, M., Ferrari, S., Uguzzoni, U., \& Natali, A. (2000). Teaching and training in the psychiatric-psychosomatic consultation-liaison setting. Psychotherapy and Psychosomatics, 69, 221-228.

Robinson, W. D., Geske, J. A., Prest, L. A., \& Barnacle, R. (2005). Depression treatment in primary care. Journal of the American Board Family Practice, 18, 79-86.

Smith, G. C. (2009). From consultation-liaison psychiatry to integrated care for multiple and complex needs. Australian and New Zealand Journal of Psychiatry, 43, 1-12.

Ustun, T. B., Ayuso-Mateos, J. L., Chatterji, S., Mathers, C., \& Murray, C. J. (2004). Global burden of depressive disorder in the year 2000. British Journal of Psychiatry, 184, 386-392.

Von Korff, M. R. (2001). Improving outcomes in depression. British Medical Journal, 323, 948-949. 